Internat. J. Math. \& Math. Sci.

Vol. 23, No. 10 (2000) 711-715

S0161171200002891

(c) Hindawi Publishing Corp.

\title{
BIORTHOGONALITY CONDITION FOR AXISYMMETRIC STOKES FLOW IN SPHERICAL GEOMETRIES
}

\author{
S. A. KHURI
}

(Received 15 September 1997)

\begin{abstract}
We derive the biorthogonality condition for axisymmetric Stokes flow in a region between two concentric spheres. This biorthogonality condition is a property satisfied by the eigenfunctions and adjoint eigenfunctions, which is needed to compute the coefficients of the eigenfunction expansion solution of the corresponding creeping flow problem.
\end{abstract}

Keywords and phrases. Eigenvalues, eigenfunctions, eigenfunction expansion, biorthogonality conditions, Stokes flow.

2000 Mathematics Subject Classification. Primary 76D07, 76D99, 35P10.

1. Introduction. Recently, the eigenfunction expansion method has been used extensively for solving problems of Stokes flow. The method leads to the development of a set of eigenfunctions, adjoint eigenfunctions, biorthogonality conditions and an algorithm for the computation of the eigenfunction expansions. This technique was first introduced by Smith [10] in his solution of the biharmonic problem governing the bending of a semi-infinite strip clamped at its side and loaded at its top edge.

The biorthogonal series expansion method was also used by Joseph [2] in his study of the free surface on the round edge of a flowing liquid filling a torsion flow viscometer and by Joseph and Sturges [3] in the steady flow induced in a rectangular cavity by the uniform translation of a covering plate or belt. Similar biorthogonal eigenfunction expansions and biorthogonality conditions are required for the axisymmetric Stokes flow problems in a wedge shaped trench studied by Liu and Joseph [7], the axisymmetric Stokes flow in a cone studied by Liu and Joseph [8] and for the problem of Stokes flow in a trench between concentric cylinders studied by Yoo and Joseph [11].

Most recently, biorthogonality conditions were used by Khuri to solve Stokes flow in a sectorial cavity [5] and by Khuri and Wang for solving Stokes flow around a bend [6].

The previous references are just a small sample of problems arising in Stokes flow and elasticity which can be solved in biorthogonal series of eigenfunctions generated by separating variables. A list of several other problems is given in $[4,9]$.

In this paper, we derive the biorthogonality condition for axisymmetric Stokes flow in a spherical region by implementing a theorem proved by Khuri [5]. This biorthogonality condition is a property satisfied by the eigenfunctions and adjoint eigenfunctions, which is needed to compute the coefficients of the eigenfunction expansion solution of the corresponding creeping flow problem. 
2. Biorthogonality conditions. We state a biorthogonality property satisfied by the eigenfunctions and adjoint eigenfunctions of the following fourth-order boundary value problem:

$$
\left(P_{0}(r) y^{\prime \prime}(r)\right)^{\prime \prime}+\left(P_{1}(r ; \alpha) y^{\prime}(r)\right)^{\prime}+P_{2}(r ; \alpha) y(r)=0 \quad r \in\left[r_{1}, r_{2}\right]
$$

The boundary conditions are given by

$$
y\left(r_{1}\right)=y\left(r_{2}\right)=y^{\prime}\left(r_{1}\right)=y^{\prime}\left(r_{2}\right)=0 .
$$

This biorthogonality condition, given in Theorem 2.1, which was proved by Khuri [5], gives the biorthogonality property for the boundary value problem given in equations (2.1) and (2.2) with certain restrictions imposed on the coefficients.

THEOREM 2.1 (biorthogonality condition). Consider the boundary value problem given in (2.1) and (2.2), where $P_{0}(r), P_{1}^{\prime \prime}(r ; \alpha), P_{2}(r ; \alpha)$ are continuous and $P_{0}(r) \neq 0$ on $r_{1} \leq r \leq r_{2} . P_{i}$ in equation (2.1) is a polynomial of degree at most $i$ in the parameter $\alpha$, in particular, let $P_{1}(r ; \alpha)=p_{11}(r) \alpha+p_{12}(r)$, and we require

$$
\begin{gathered}
P_{1}^{2}(r ; \alpha)-4 P_{0}(r) P_{2}(r ; \alpha)=p_{31}(r) \alpha+p_{32}(r), \\
p_{11}^{2}(r)+p_{31}^{2}(r) \neq 0 .
\end{gathered}
$$

Then with $P_{n}^{*}$ defined by

$$
P_{n}^{*}=\int_{r_{1}}^{r_{2}}\left[\phi_{2}^{(n)}(r), \phi_{1}^{(n)}(r)\right] B(r)\left[\begin{array}{l}
\phi_{1}^{(n)}(r) \\
\phi_{2}^{(n)}(r)
\end{array}\right] d r,
$$

we have the following biorthogonality condition:

$$
\int_{r_{1}}^{r_{2}}\left[\phi_{2}^{(m)}(r), \phi_{1}^{(m)}(r)\right] B(r)\left[\begin{array}{l}
\phi_{1}^{(n)}(r) \\
\phi_{2}^{(n)}(r)
\end{array}\right] d r=P_{n}^{*} \delta_{m n},
$$

where $\delta_{m n}$ is the Kronecker's delta,

$$
B(r)=\left(\begin{array}{cc}
-\frac{1}{2} \frac{p_{11}(r)}{P_{0}(r)} & 0 \\
\frac{1}{2} p_{11}^{\prime \prime}(r)+\frac{1}{4} \frac{p_{31}(r)}{P_{0}(r)} & -\frac{1}{2} \frac{p_{11}(r)}{P_{0}(r)}
\end{array}\right)
$$

with

$$
\begin{gathered}
\phi_{1}^{(n)}(r)=y_{n}(r), \\
\phi_{2}^{(n)}(r)=P_{0}(r) y_{n}^{\prime \prime}(r)+\frac{1}{2} P_{1}\left(r ; \alpha_{n}\right) y_{n}(r) .
\end{gathered}
$$

Here $y_{i}$ is an eigenfunction of equation (2.1) corresponding to the eigenvalue $\alpha_{i}$. Assume the eigenvalues $\alpha_{i}$ are simple. 
3. Axisymmetric Stokes flow in spherical regions. In this section, the biorthogonality condition for the axisymmetrical creeping flow in a region between two concentric spheres is derived. The flow region is

$$
v=\left\{r, \theta: 0<r_{1} \leq r \leq r_{2},-\theta_{1} \leq \theta \leq \theta_{1}\right\}
$$

The Stokes flow equation in spherical coordinates $(r, \theta, \phi)$ in $v$ is given by

$$
E^{4} \Psi(r, \theta)=\left(\frac{\partial^{2}}{\partial r^{2}}+\frac{1}{r^{2}} \frac{\partial^{2}}{\partial \theta^{2}}-\frac{\cot \theta}{r^{2}} \frac{\partial}{\partial \theta}\right)^{2} \Psi(r, \theta)=0 .
$$

The velocity components in the $(r, \theta)$ direction in terms of the stream function are given by

$$
v_{r}=-\frac{1}{r^{2} \sin \theta} \frac{\partial \Psi}{\partial \theta}, \quad v_{\theta}=\frac{1}{r \sin \theta} \frac{\partial \Psi}{\partial r} .
$$

Requiring the velocity to vanish on $r=r_{1}, r_{2}$, (3.3) gives

$$
\Psi\left(r_{1}, \theta\right)=\Psi\left(r_{2}, \theta\right)=\frac{\partial \Psi}{\partial r}\left(r_{1}, \theta\right)=\frac{\partial \Psi}{\partial r}\left(r_{2}, \theta\right)=0 .
$$

Separable solutions of (3.2) and (3.4) in the form

$$
\Psi(r, \theta) \sim T(\cos \theta) y(r)
$$

exist (see [1]) when $y(r)$ satisfies the following equation:

$$
y^{(4)}+\frac{2}{r^{2}} p(1-p) y^{(2)}-\frac{4}{r^{3}} p(1-p) y^{(1)}+p(1-p)(2+p)(3-p) \frac{1}{r^{4}} y=0
$$

and the boundary conditions

$$
y\left(r_{1}\right)=y\left(r_{2}\right)=y^{\prime}\left(r_{1}\right)=y^{\prime}\left(r_{2}\right)=0 .
$$

Seeking an eigenfunction solution in $r$ direction it is necessary that the function $T(\eta)$, where $\eta=\cos \theta$ be required to satisfy the following equation:

$$
\left(1-\eta^{2}\right) T^{\prime \prime}(\eta)-p(1-p) T(\eta)=0 .
$$

Equation (3.8) is Gegenbauer's equation of degree $-1 / 2$ where $p$ could be complex. The two independent solutions of (3.8) are $C_{p}^{-1 / 2}(\eta)$ and $D_{p}^{-1 / 2}(\eta)$ that are termed as Gegenbauer functions of the first and second kind, respectively. Clearly, equation (3.6) can be written in the following form:

$$
\left(y^{\prime \prime}\right)^{\prime \prime}+2 p(1-p)\left(\frac{1}{r^{2}} y^{\prime}\right)^{\prime}+p(1-p)(2+p)(3-p) \frac{1}{r^{4}} y=0 .
$$

The hypothesis of Theorem 2.1 is satisfied when $\alpha_{n} \neq \alpha_{m}$ with

$$
P_{0}(r)=1 ; \quad P_{1}(r ; \alpha)=\frac{2}{r^{2}} \alpha ; \quad P_{2}(r ; \alpha)=\frac{1}{r^{4}} \alpha(\alpha+6),
$$


where

$$
\alpha=p(1-p)
$$

Since

$$
P_{1}^{2}(r ; \alpha)-4 P_{0}(r) P_{2}(r ; \alpha)=-\frac{24}{r^{4}} \alpha
$$

so

$$
p_{31}(r)=-\frac{24}{r^{4}} ; \quad p_{32}(r)=0
$$

Clearly,

$$
p_{11}(r)=\frac{2}{r^{2}} ; \quad p_{12}(r)=0
$$

Thus using Theorem 2.1. The biorthogonality condition is given by

$$
\int_{r_{1}}^{r_{2}} \frac{-1}{r^{2}}\left[\psi_{1}^{(m)}(r), \psi_{2}^{(m)}(r)\right]\left[\begin{array}{l}
\phi_{1}^{(n)}(r) \\
\phi_{2}^{(n)}(r)
\end{array}\right] d r=P_{n}^{*} \delta_{m n}, \quad p_{n}\left(1-p_{n}\right) \neq p_{m}\left(1-p_{m}\right)
$$

upon using

$$
B(r)=-\frac{1}{r^{2}} I_{2 \times 2}=\left(\begin{array}{cc}
-\frac{1}{r^{2}} & 0 \\
0 & -\frac{1}{r^{2}}
\end{array}\right),
$$

where $I_{2 \times 2}$ is the identity matrix. The eigenfunctions satisfy

$$
\phi_{1}^{(n)}(r)=y_{n}(r), \quad \phi_{2}^{(n)}(r)=y_{n}^{\prime \prime}(r)+\frac{\alpha_{n}}{r^{2}} y_{n}(r)
$$

and the adjoint eigenfunctions satisfy

$$
\psi_{1}^{(m)}(r)=y_{m}^{\prime \prime}(r)+\frac{\alpha_{m}}{r^{2}} y_{m}(r), \quad \psi_{2}^{(m)}(r)=y_{m}(r),
$$

where

$$
\alpha_{n}=p_{n}\left(1-p_{n}\right)
$$

\section{REFERENCES}

[1] J. Happel and H. Brenner, Low Reynolds Number Hydrodynamics with Special Applications to Particulate Media, Prentice-Hall Inc., Englewood Cliffs, N.J., 1965. MR 33\#3562.

[2] D. D. Joseph, Slow motion and viscometric motion; stability and bifurcation of the rest state of a simple fluid, Arch. Rational Mech. Anal. 56 (1974/75), 99-157. MR 50\#3744. Zbl 295.76035. 
[3] D. D. Joseph and L. Sturges, The convergence of biorthogonal series for biharmonic and Stokes flow edge problems. II, SIAM J. Appl. Math. 34 (1978), no. 1, 7-26. MR 57\#14765. Zbl 379.31001.

[4] D. D. Joseph, L. D. Sturges, and W. H. Warner, Convergence of biorthogonal series of biharmonic eigenfunctions by the method of Titchmarsh, Arch. Rational Mech. Anal. 78 (1982), no. 3, 223-274. MR 84i:35108. Zbl 486.35061.

[5] S. A. Khuri, Biorthogonal series solution of Stokes flow problems in sectorial regions, SIAM J. Appl. Math. 56 (1996), no. 1, 19-39. MR 96i:76035. Zbl 844.76019.

[6] S. A. Khuri and C. Y. Wang, Stokes flow around a bend, Quart. Appl. Math. 55 (1997), no. 3, 573-600. MR 98d:76042. Zbl 887.76020.

[7] C. H. Liu and D. D. Joseph, Stokes flow in wedge-shaped trenches, J. Fluid Mech. 80 (1977), 443-463. Zbl 355.76027.

[8] _ Stokes flow in conical trenches, SIAM J. Appl. Math. 34 (1978), no. 2, 286-296. MR 80d:76034. Zbl 376.76018.

[9] T. N. Phillips, Singular matched eigenfunction expansions for Stokes flow around a corner, IMA J. Appl. Math. 42 (1989), no. 1, 13-26. MR 90f:76044. Zbl 674.76020.

[10] R. C. T. Smith, The bending of a semi-infinite strip, Australian J. Sci. Research. Ser. A. 5 (1952), 227-237. MR 15,842a.

[11] J. Y. Yoo and D. D. Joseph, Stokes flow in a trench between concentric cylinders, SIAM J. Appl. Math. 34 (1978), no. 2, 247-285. MR 80d:76033. Zbl 376.76019.

KHURI: DEPARTMENT OF COMPUTER SCIENCE, MATHEMATICS AND STATISTICS, AMERICAN UNIVERSITY OF SHARJAH, P.O. BOX 26666-739, SHARJAH, UNITED ARAB EMIRATES.

E-mail address: skhoury@aus.ac.ae 


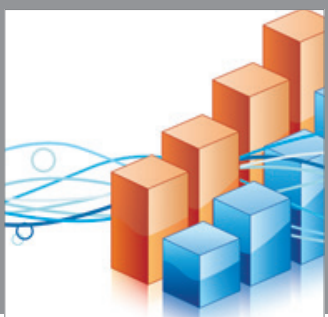

Advances in

Operations Research

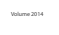

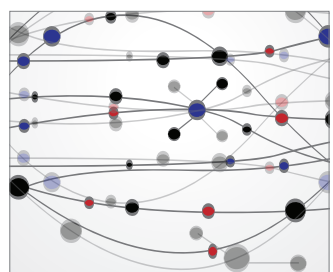

\section{The Scientific} World Journal
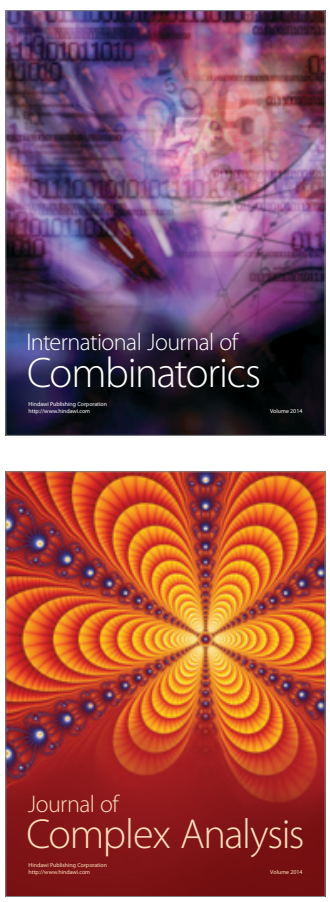

International Journal of

Mathematics and

Mathematical

Sciences
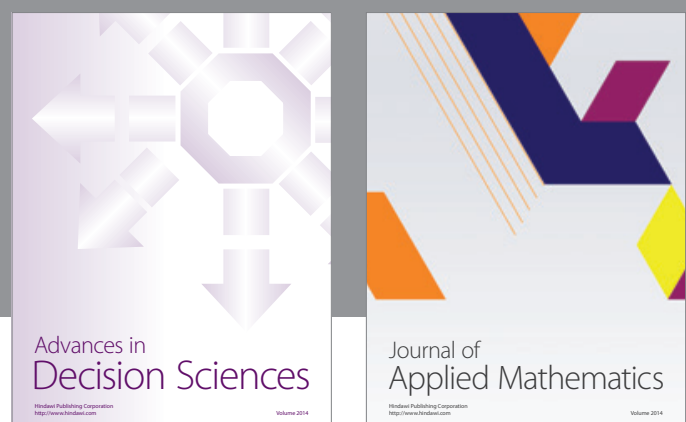

Journal of

Applied Mathematics
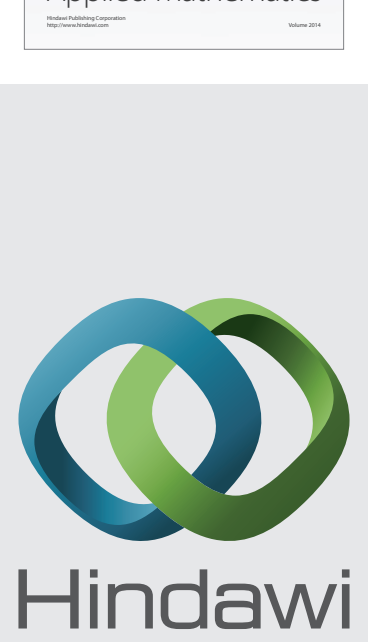

Submit your manuscripts at http://www.hindawi.com
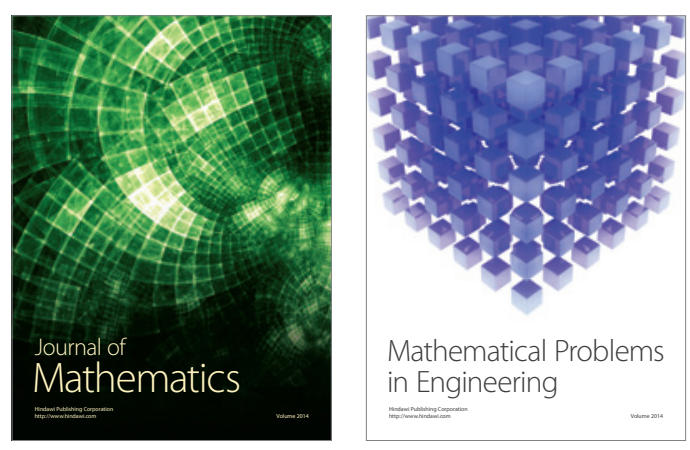

Mathematical Problems in Engineering
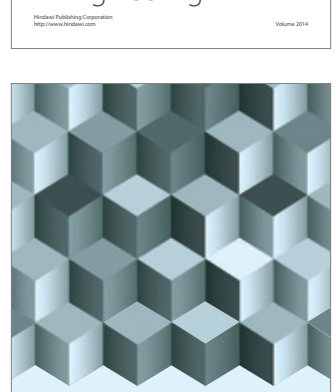

Journal of

Function Spaces
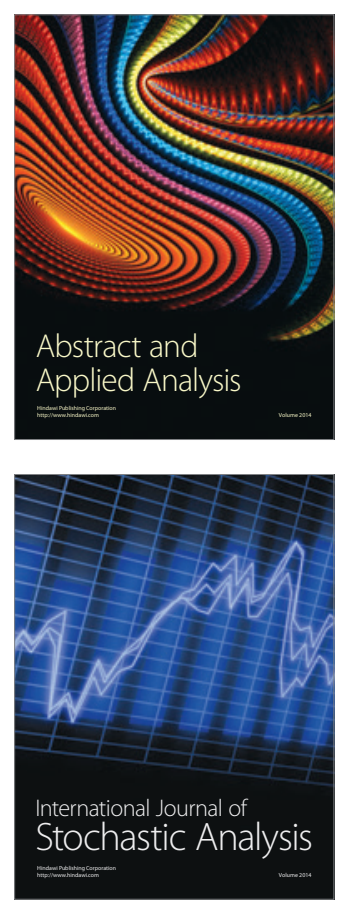

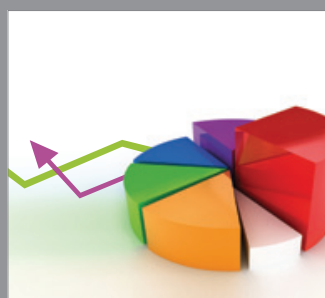

ournal of

Probability and Statistics

Promensencen
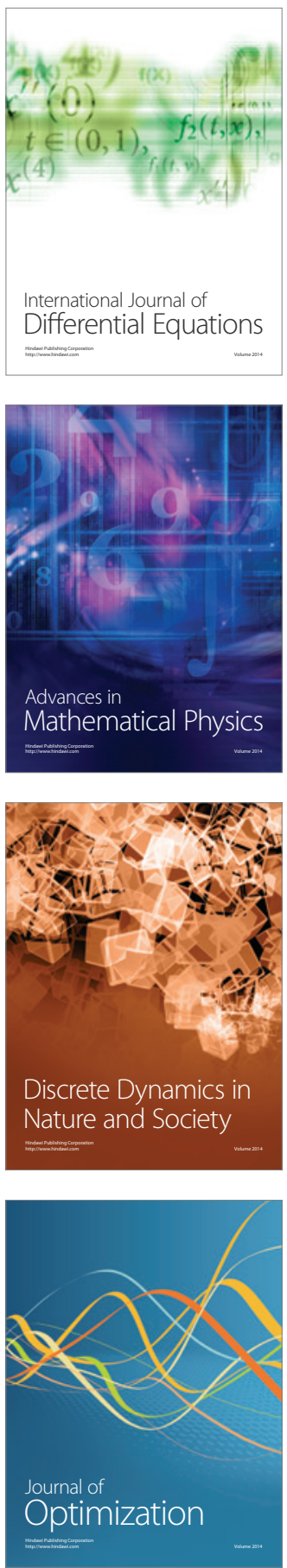